Delft University of Technology

\title{
Three Categories of Context-Aware Systems
}

Shishkov, Boris; Larsen, John Bruntse; Warnier, Martijn; Janssen, Marijn

DOI

10.1007/978-3-319-94214-8_12

Publication date

2018

Document Version

Final published version

Published in

Proceedings of Business Modeling and Software Design - 8th International Symposium, BMSD 2018

\section{Citation (APA)}

Shishkov, B., Larsen, J. B., Warnier, M., \& Janssen, M. (2018). Three Categories of Context-Aware

Systems. In Proceedings of Business Modeling and Software Design - 8th International Symposium, BMSD 2018 (Vol. 319, pp. 185-202). (Lecture Notes in Business Information Processing; Vol. 319). Springer Science+Business Media. https://doi.org/10.1007/978-3-319-94214-8_12

\section{Important note}

To cite this publication, please use the final published version (if applicable).

Please check the document version above.

\section{Copyright}

Other than for strictly personal use, it is not permitted to download, forward or distribute the text or part of it, without the consent of the author(s) and/or copyright holder(s), unless the work is under an open content license such as Creative Commons.

Takedown policy

Please contact us and provide details if you believe this document breaches copyrights.

We will remove access to the work immediately and investigate your claim. 
Green Open Access added to TU Delft Institutional Repository

'You share, we take care!' - Taverne project

https://www.openaccess.nl/en/you-share-we-take-care

Otherwise as indicated in the copyright section: the publisher is the copyright holder of this work and the author uses the Dutch legislation to make this work public. 


\title{
Three Categories of Context-Aware Systems
}

\author{
Boris Shishkov ${ }^{1,4(凶)}$, John Bruntse Larsen², Martijn Warnier ${ }^{3}$, \\ and Marijn Janssen ${ }^{3}$ \\ ${ }^{1}$ Institute of Mathematics and Informatics, \\ Bulgarian Academy of Sciences, Sofia, Bulgaria \\ b.b.shishkov@iicrest.org \\ ${ }^{2}$ Department of Applied Mathematics and Computer Science, \\ Technical University of Denmark, Lyngby, Denmark \\ jobla@dtu.dk \\ ${ }^{3}$ Faculty of Technology, Policy, and Management, \\ Delft University of Technology, Delft, The Netherlands \\ \{M. E. Warnier, M. F.W.H. A. Janssen\}@tudelft.nl \\ ${ }^{4}$ Institute IICREST, Sofia, Bulgaria
}

\begin{abstract}
With regard to context-aware systems: some optimize systeminternal processes, based on the context state at hand; others maximize the user-perceived effectiveness of delivered services, by providing different service variants depending on the situation of the user; still others are about offering value-sensitivity when the society demands so. Even though those three perspectives cover a broad range of currently relevant applications there are no widely accepted and commonly used corresponding concepts and terms. This is an obstacle to broadly understand, effectively integrate, and adequately assess such systems. We address this problem, by considering a (component-based) methodological derivation of technical (software) specifications based on underlying enterprise models. That is because context states are about the enterprise environment of a (software) system while the delivery of context-aware services is about technical (software) functionalities; hence, we need a perspective on both. We consider the SDBC (Software Derived from Business Components) approach that brings together enterprise modeling and software specification. On that basis: (a) We deliver a base context-awareness conceptualization; (b) We partially align it to agent technology because adapting behaviors to environments assumes some kind of pro-activity that is only fully covered by agent systems, in our view. We partially illustrate our proposed conceptualization and particularly - the agent technology implications, by means of a case example featuring land border security.
\end{abstract}

Keywords: Modeling $\cdot$ System design $\cdot$ Context-awareness $\cdot$ SDBC AORTA

\section{Introduction}

Whenever a group of entities collectively realize a goal, we consider them to belong to a system [17]. An adaptable system has the ability to adjust to new conditions [8]. We argue that an essential feature of adaptable systems is context-awareness 
[22] - this is adjusting the system behavior depending on the situation at hand (context state). Our observation is that context-aware systems are all about adjusting "something" to the context state; however, what is adjusted differs: (i) Some context-aware systems optimize system-internal processes based on the context state at hand [3, 12], for example: regulating the electro-consumption of home appliances for the sake of keeping the overall building consumption within some boundaries. (ii) Other context-aware systems maximize the user-perceived effectiveness of delivered services, by providing different service variants depending on the situation of the user [1], for example: treating a distantly monitored patient in one way when his/her condition is normal and in another way in case of emergency. (iii) Still other context-aware systems are about offering value-sensitivity when the society demands so [7], for example: in the case of supporting judiciary processes, different levels of transparency are to be provided to different categories of stakeholders. Not claiming exhaustiveness, we argue that those three context-awareness perspectives cover a broad range of currently relevant applications, while corresponding system categorizations are observed to be missing in literature, especially as it concerns real-life (business) processes. Hence, we propose and elaborate (in the following section) the above categorization.

Further, there are no widely accepted and commonly used concepts and terms with respect to (i), (ii), and (iii). This is considered to be an obstacle with regard to broadly understanding, effectively integrating, and adequately assessing such systems. We address this problem, by taking a (component-based-) technical/software design perspective, assuming a desired methodological derivation of the technical (software) specifications on the basis of underlying enterprise models. That is because context states are about the enterprise environment of a (software) system while the delivery of context-aware services is about technical (software) functionalities; hence, we need a perspective on both. Stepping on previous work, we consider the SDBC (Software Derived from Business Components) approach that brings together enterprise modeling and software specification [17, 18, 20]. On that basis:

- We deliver a base context-awareness conceptualization (inspired by the current discussion and the analysis carried out in Sect. 2) that is claimed to hold for all (i), (ii), and (iii).

- We partially align that conceptualization to agent technology [11, 27] because adapting behaviors to environments is considered to assume some kind of pro-activity that is only fully covered by agent systems, in our view.

We partially illustrate our proposed conceptualization and particularly - the agent technology implications, by means of a case example featuring land border security.

The remaining of the current paper is organized as follows: Sect. 2 elaborates and discusses the above-mentioned context-awareness perspectives (optimizing internal processes, maximizing the user-perceived effectiveness, being value-sensitive). In Sect. 3, we: (a) briefly outline the SDBC approach that gives the general methodological guidelines we follow; (b) elicit (in line with SDBC) the base contextawareness system concepts, referring to the analysis in Sect. 2; (c) enrich those concepts from the AORTA (agent-technology) perspective; (d) distill on that basis a meta-model that is considered a key contribution of this paper and propose guidelines 
refinements. Assuming the SDBC methodological guidelines and referring to a real-life case study that is featuring the usage of drones in support of land border security, we partially illustrate in Sect. 4 the meta-model and the AORTA framework influence, emphasizing the applicability with regard to all three categories of context-aware systems, as discussed above. We justify the adequacy of the delivered contribution in Sect. 5, by analyzing related work. Finally, in Sect. 6 we conclude the paper.

\section{System Behavior Perspectives}

Referring to the notions considered in the Introduction, we will firstly elaborate on the context-driven optimization of system-internal processes (Subsect. 2.1), secondly - on the context-driven maximization of the user-perceived effectiveness (Subsect. 2.2), and finally, on the context-driven value-sensitivity (Subsect. 2.3). It is often that the context-awareness is enabled by sensor technology [21] allowing to "know" what is happening around; alternatively, there should be other ways of "sensing" the environment [1]. As it concerns (i), (ii), and (iii) - see the previous section - this counts for all of them. Further, considering the essence of their underlying system behaviors, we use the following labels: SELF-MANAGING CONTEXT-AWARE SYSTEM for (i); USER-DRIVEN CONTEXT-AWARE SYSTEM for (ii), and VALUE-SENSITIVE CONTEXT-AWARE SYSTEM for (iii). Finally, even though most often contextaware systems are "sensitive" to changes in the system environment, it is also possible that the "sensitivity" is towards internal issues (things happening inside the system (not in the environment) may trigger either internal optimizations, or changes in the services delivered to the user, or a reconsideration of the "covered" values). In this paper, we are not restrictive with regard to the "sensitivity" (whether it concerns the system or the environment).

\subsection{Self-Managing Context-Aware Systems (SMCAS)}

SMCAS' context-awareness is directed towards internal (system) optimization purposes [16]. Such autonomic solutions [12] are proposed as a way to reduce the cost of maintaining complex systems, and to increase the human ability to manage these systems properly, by automating (part of) their working. In essence, self-managing system can be characterized by a feedback 10op mechanism that allows them to optimize their working based on input from the environment. For the basic feedback loop, the system receives input from the environment (monitor) and can change its behavior which in turn has an effect on the environment (effector). In Autonomic Computing [10] this basic loop is extended into four components, resulting in the MAPE Cycle, see Fig. 1 (left). Next to the "monitor" and "effector" phases, the system internally has an analyze phase that processes environmental input and a plan phase that changes the internal and external working of the system.

Taking this one step further, an autonomic system can manage another system (the managed system) by placing it in the MAPE Cycle as shown in Fig. 1 (right). Placed in such a configuration, the autonomic and managed systems together form a SELF-MANAGING SYSTEM or self-adaptive system [14]. 

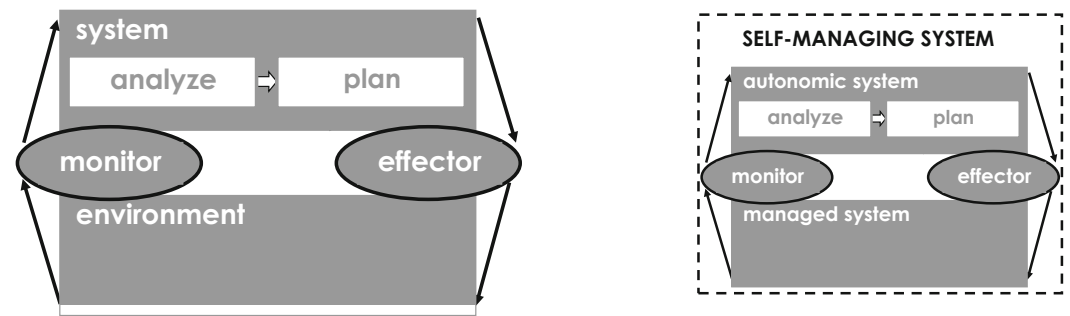

Fig. 1. Monitor-Analyze-Plan-Effect (MAPE) cycle (left); A managed system and an autonomic system that together form a self-managing system (right)

Internally self-managing systems optimize their behavior based on inputs to and outputs from the managed system. Such state updates can range from simple if-then rules (for example: if the temperature is below zero degrees, then pre-heat the car) to more sophisticated approaches such as those based on machine learning techniques (e.g., neural networks or inference engines that determine the best action based on a large internal knowledge base). Thus, the main objective of self-managing systems is to optimize their internal working based on inputs from the environments.

\subsection{User-Driven Context-Aware Systems (UDCAS)}

The UDCAS' context-awareness is directed towards the maximization of the external (user) satisfaction [1]. Hence, such systems should be able to: (i) identify the situation of the user (possibly through sensors); (ii) deliver a service to the user, that is suited for the particular situation, as illustrated in Fig. 2 (left).

As it is seen from the figure, a service is delivered to the user and the user is considered within his or her context, such that the service is adapted on the basis of the context state (or situation) the user finds himself/ herself in. That state is to be somehow sensed and often technical devices, such as sensors, are used for this purpose. UDCAS actually deliver services to the user by means of ICT (Information and Communication Technology) applications [17] (applications, for short). Hence, unlike "traditional" applications assuming that users would have common requirements independent of their context, user-driven context-aware applications are capable of adapting their behavior to the situation of the user (this is especially relevant to services delivered via mobile devices). Hence, such applications are, to a greater or lesser extent, aware of the user context situation (for example, user is at home, user is traveling) and provide the desirable services corresponding to the situation at hand. This quality points also to another related characteristic, namely that user-driven context-aware applications must be able to capture or be informed about information on the context of users, preferably without effort and conscious acts from the user part [18]. Hence, a basic assumption underlying the development of user-driven context-aware applications is that user needs are not static, however partially dependent on the particular situation the user finds himself/herself in, as already mentioned. For example, depending on his/her current location, time, activity, social environment, environmental properties, 
or physiological properties, the user may have different interests, preferences, or needs with respect to the services that can be provided by applications.

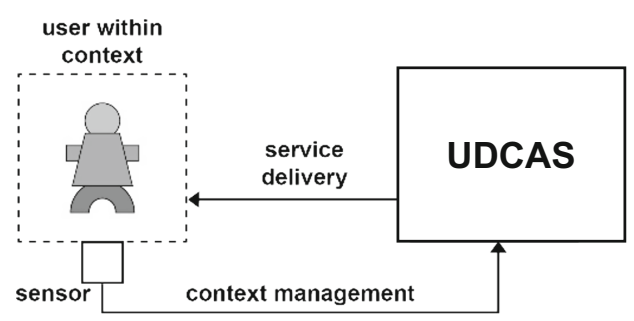

\section{USER-DRIVEN SYSTEM}

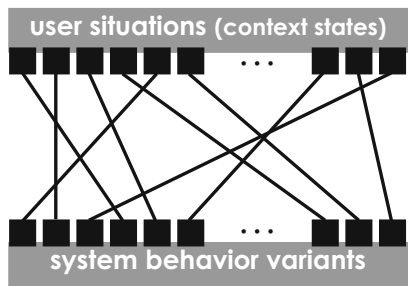

Fig. 2. The UDCAS vision (inspired by [18, 22]): a schematic representation (left); Contextstates-driven system behavior variants (right)

User-driven context-aware applications are thus primarily motivated by their potential to increase the user-perceived effectiveness, i.e. to provide services that better suit the needs of the user, by taking account of the user situation. We refer to the collection of parameters that determine the situation of a user, and which are relevant for the application in pursue of user-perceived effectiveness, as user context, or context for short, in accordance to definitions found in literature [5].

Finally, UDCAS are hence about delivering behavior variants to corresponding user situations (context states), as illustrated by Fig. 2 (right). The idea is that for each context state, the system has a behavior variant. Nevertheless, this would not be always realistic because if the possible context states are too many (for example: tens or hundreds), the effort for "preparing" (at design time) behavior variants would be huge. For this reason, statistical data analysis and probability studies are needed, as studied in [17], for establishing the context states of high occurrence probability (for example, during a normal working day, an employee would most probably be either at work or at home, or traveling, and it is not very likely that the employee is somewhere else, especially during day hours). Hence all other (possibly hundreds) low probability context states would not need to be addressed at design time; instead, a "collecting" context state (for example, labelled: OTHER) may be considered at design time, assuming a more generic behavior algorithm, such that the behavior is "tuned" at real time (possibly in a rule-based way). This would of course lead to lower quality-of-service which is nevertheless justified since it would be very rare that the OTHER behavior variant is triggered. Thus, this is a matter of "trade-off" between quality-of-service and resources.

\subsection{Value-Sensitive Context-Aware Systems (VSCAS)}

VSCAS' context-awareness is directed towards a sensitivity to public values [7]. Public values (values for short) like privacy and data protection, security, accountability, integrity and provenance, and sustainable data storage, often need to be incorporated in the functionalities of information systems. Hence, the first question to be answered is: How do values relate to requirements (we mean particularly 
non-functional requirements because values are essentially non-functional)? In answering this question, we refer to [19] who argue that values are desires of the general public (or public institutions/organizations that claim to represent the general public), that are about properties considered societally valuable, such as respecting the privacy of citizens or prohibiting polluting activities. Even though values are to be broadly accepted (that is why they are public), they may concern individuals (for example: considering privacy). Hence, put broadly, values concern the societal expectations with regard to the way services should be delivered and with regard to the above question: values are desires or goals, not requirements. Values are abstract and not directly related to an enterprise or software system, as opposed to requirements. Moreover, values are construct by and for society and not by and for the enterprise domain in which a specific system will be used. Those domains may overlap but are not the same. Values that are adopted as goals by an enterprise would therefore impact the requirements on a system that the enterprise wants to introduce in order to realize its goals. For this reason, the impact of values cannot be limited to non-functional requirements. It is therefore considered important to clearly distinguish values from requirements and acknowledge the limitations of requirements engineering with regard to the development of value-sensitive (software) systems.

Hence, the development of systems should take into account the objective, the user needs, but also the operating and societal context. In this way values can be used as a guidance for making choices when developing systems; looking at possible tensions among values is an issue as well. Thus, we have identified challenges in several directions:

Firstly, values are normative by nature and different stakeholders might prefer different values; also, values might differ among countries and cultures.

Secondly, even though different societies may agree on a value at high-level, their cultural and other differences may "push" for different value "realizations". This may result in different operationalizations and implementations of the same value.

Thirdly, values may be conflicting to each other (for example: fulfilling two values at the same time may be impossible). Searching for criminals might lead to violating the privacy of innocent people, for example.

Thus, in considering VSCAS, it is important to be aware that different context states may assume the consideration of different values, which in turn would mean different system functionality variants. Nevertheless, this goes beyond a mapping just between values and non-functional requirements and would assume a broader consideration of the software functionalities specification.

Overall: computing power becomes larger, wireless telecommunications are advancing, and sensor technology is developing fast [17]; this allows for ubiquitous network connectivity and numerous capabilities of smart devices, as a basis for developments in several directions: (a) Systems that are traditionally designed for one specific situation and task can be augmented to become "smarter", being able to operate in complex environments. (b) Systems are empowered to "sense" what is going on inside them and also what is going on with the end user while (s) he is utilizing corresponding services. This concerns the system-internal processes, the way services are delivered to users, and the way values are considered, as discussed in the current section. In the following section, we will present our proposed way of modeling contextaware systems, taking into account those three system behavior perspectives. 


\section{Proposed Modeling and Design}

Furthering previous work, we consider SDBC (see Sect. 1) as the approach of choice (generally) because of several reasons: its strengths in aligning enterprise modeling and software specifications, its component-orientation and support for re-use, and its previous use for specifying context-aware and privacy-sensitive systems [17, 18, 20]. We will thus briefly introduce SDBC in this section. We will then present the main concepts we consider, making sure that they are consistent with SDBC and relevant to the three categories of context-aware systems, as considered in the previous section; we will also provide a conceptual enrichment from an agent-technology perspective (see Sect. 1). We will then reflect this in a meta-model derived accordingly. And in the end, we will narrow the design scope (in the above context) to only touch upon issues that concern the key features of the considered three categories of context-aware systems.

\subsection{SDBC}

$S D B C$ is an approach (consistent with MDA [15]) that is focused on the derivation of software specification models on the basis of corresponding (re-usable) enterprise models. SDBC is based on three key ideas: (i) The software system-to-be is considered in its enterprise context, which means that the software specification models are to stem from corresponding enterprise models; this means in turn that a deep understanding is needed on real-life (enterprise-level) processes, corresponding roles, behavior patterns, and so on. (ii) By bringing together two disciplines (enterprise engineering and software engineering), SDBC pushes for applying social theories in addressing enterprise-engineering-related tasks and for applying computing paradigms in addressing software-engineering-related tasks, and also for integrating the two, by means of sound methodological guidelines. (iii) Acknowledging the value of re-use in current software development, SDBC pushes for the identification of re-usable (generic) enterprise engineering building blocks whose models could be reflected accordingly in corresponding software specification models. We refer to [17] for information on SDBC and we are reflecting the SDBC outline in Fig. 3.

As the figure suggests, there are two SDBC modeling milestones, namely enterprise modeling (first milestone) and software specification (second milestone). The first milestone has as input a case briefing (the initial (textual) information based on which the software development is to start) and the so called domain-imposed requirements (those are the domain regulations to which the software system-to-be should conform). Based on such an input, an analysis should follow, aiming at structuring the information, identifying missing information, and so on. This is to be followed by the identification (supported by corresponding social theories) of enterprise modeling entities and their inter-relations. Then, the causalities concerning those inter-relations need to be modeled, such that we know what is required in order for something else to happen [23]. On that basis, the dynamics (the entities' behavior) is to be considered, featured by transactions [17]. This all leads to the creation of enterprise models that are elaborated in terms of composition, structure, and dynamics (all this pointing also to corresponding data aspects) - they could either "feed" further software specifications and/or be "stored" for further use by enterprise engineers. Such enterprise models could possibly be reflected in corresponding business 


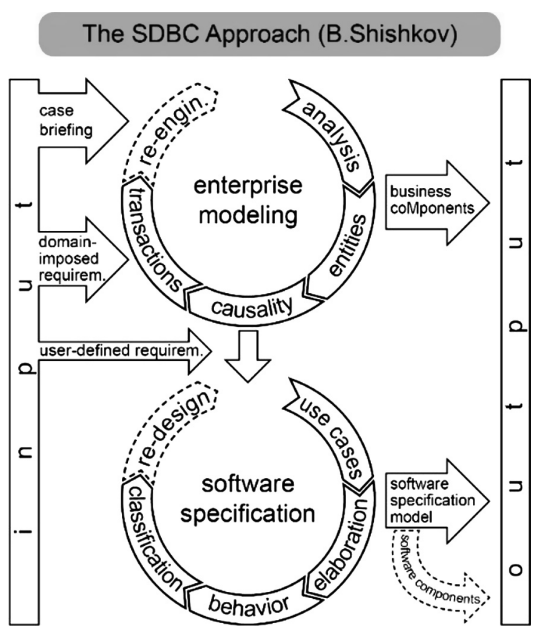

Fig. 3. SDBC - outline (Source: [20], p. 48)

components (models of business components [17]). Next to that, re-visiting such models could possibly inspire enterprise re-design activities, as shown in Fig. 3.

Furthermore, the second milestone uses as input the enterprise model (see above) and the so called user-defined requirements (those requirements reflect the demands of the (future) users of the software system-to-be towards its functioning).

That input "feeds" the derivation of a use case model featuring the software system-to-be. Such a software specification starting point is not only consistent with the Rational Unified Process - RUP [13] and the Unified Modeling Language - UML [25] but is also considered to be broadly accepted beyond RUP-UML [17]. The use cases are then elaborated, inspired by studies of Cockburn [4] and Shishkov [17], such that software behavior models and classification can be derived accordingly. The output is a software specification model adequately elaborated in terms of statics and dynamics. Applying de-composition, such a model can be reflected in corresponding software components, as shown in the figure. Such an output could be inspiration for proposing in the future software re-designs, possibly addressing new requirements.

Further, in bringing together the first milestone of SDBC and the second one, we need to be aware of possible granularity mismatches. The enterprise modeling is featuring business processes and corresponding business coMponents but this is not necessarily the level of granularity concerning the software components of the system-to-be. With this in mind, an ICT application is considered as matching the granularity level of a business component - an ICT application is an implemented software product realizing a particular functionality for the benefit of entities that are part of the composition of an enterprise system and/or a (corresponding) enterprise information system. Thus the label software specification model, as presented in Fig. 3, corresponds to a particular ICT application being specified. Software components in turn are viewed as implemented pieces of software, which represent parts of an ICT application, and which collaborate among each other driven by the goal of realizing the functionality of the application (functionally, a software component is a part of an ICT 
application, which is self-contained, customizable, and composable, possessing a clearly defined function and interfaces to the other parts of the application, and which can also be deployed independently). Hence: a software component is a conceptual specification model of a software component; the second SDBC milestone is about the identification of software coMponents and corresponding software components.

\subsection{Conceptualization}

In this sub-section, we will firstly consider the basic concepts aligned with SDBC and the system behavior perspectives, as considered in the previous section.

\section{BASIC CONCEPTS (GENERAL)}

SYSTEM: A collection of elements possibly interacting with each other, driven by the purpose of delivering a service to another entity or group of entities.

SUB-SYSTEM: A system part identified based on a functional decomposition with regard to the system (hence, a system can optimize itself, by optimizing corresponding sub-systems).

ENVIRONMENT: Anything not belonging to a system belongs to the system environment Part of the environment concerns those environmental entities that are assumed to have some interaction with the system.

ENTITIES: Composition elements with regard to a system / environment

- Human vs Artificial entities;

- Passive (a passive entity is an entity that only performs actions when another entity interacts with it) vs Autonomous (an autonomous entity is an entity that performs actions on its own initiative) entities;

- Sensing (capturing context data in support of the system's service delivery) vs Actuator (causing changes in the environment on behalf of the system) entities.

ROLE: The state of carrying out certain objectives

- The entity-role combination is labelled "actor-role".

ACTOR: An autonomous entity that can enact a role.

USER: An actor that the system services.

ACTION: Something done by an entity

- A sequence of actions, occurring between two entities that are collaborating in support of the service delivery, is labelled "inter-action".

OBJECTIVE: The motive behind the service delivery.

REGULATIONS: Reflection of the existing norms that have impact on the service delivery, prescribing what is allowed in some situations, forbidden in others, and so on.

VALUES: Reflection of the public perception towards what is important regarding the service delivered by the systems*

* We address values that are shared among environmental human entities.

\section{BASIC CONCEPTS (FEATURING SYSTEM ADAPTATION DIMENSIONS)}

SELF-MANAGING BEHAVIOR: Context-driven enforcement of syst.-internal optimizations. USER-DRIVEN BEHAVIOR: Context-driven service adaptation based on the user situation.

VALUE-SENSITIVITY: Service adaptation inspired by values, delivered through the operationalization of values. 
Wishing to enrich those concepts from an agent-technology perspective (see the Introduction) AORTA is partially considered as a meta-model for enabling agents to perform organizational reasoning based on the OperA model [6] for agent organizations [11]. Organizational reasoning enables an agent to identify the objectives it is expected to solve, the other agents that it depends on for solving those objective, and whether an action is permitted, obliged, allowed or forbidden. In doing so the social expectations are made explicit through the AORTA concepts, which is considered an advantage when creating and reiterating the design of agents. Those concepts are shown below in the form of logical predicates:

\begin{tabular}{|ll|}
\hline CONCEPT & \multicolumn{1}{c}{ MEANING } \\
\hline role(Role, Objs $)$ & $\begin{array}{l}\text { Role is the name of a role and Objs is a set of main objec- } \\
\text { tives of that role. }\end{array}$ \\
\hline obj(Obj, SubObjs $)$ & $\begin{array}{l}\text { Obj is an objective that has SubObjs as a set of sub- } \\
\text { objectives. }\end{array}$ \\
\hline dep(Role_1, Role_2, Obj) & Role_1 depends on Role_2 in order to complete Obj. \\
\hline rea(Ag, Role) & Agent Ag enacts Role. \\
\hline cond(Role,Obj,Deadline,Cond) & $\begin{array}{l}\text { When the condition Cond holds, Role is obliged to com- } \\
\text { plete Obj before the objective Deadline. }\end{array}$ \\
\hline obl(Ag, Role, Obj, Deadline) & $\begin{array}{l}\text { Agent Ag is obliged to enact Role to complete Obj before } \\
\text { Deadline. }\end{array}$ \\
\hline $\operatorname{viol}(\mathrm{Ag}$, Role, Obj) & $\begin{array}{l}\text { Agent Ag enacting Role has violated the obligation to } \\
\text { complete Obj. }\end{array}$
\end{tabular}

A role defines a role in the organization, and the primary objectives associated with that role. By enacting a role, an agent announces to other agents in the organization that it takes upon itself that role and thus that other agents can expect it to work towards solving the objectives associated with that role. It is also possible to make an explicit notion for how an objective can be decomposed into sub-objectives, and how a role may depend on other roles to solve an objective. Finally, normative statements are supported that define conditions on how agents should solve an objective in a certain context.

AORTA divides organizational reasoning into three phases: obligation check (OC), option generation (OPG) and action execution (AE). In the OC-phase the agent uses its beliefs to check if obligations are activated, satisfied or violated, and updates its beliefs accordingly. Following that it generates possible organizational options in 
the OPG-phase, which it then considers in the AE-phase when deliberating an action. Figure 4 visualizes such a reasoning "component".

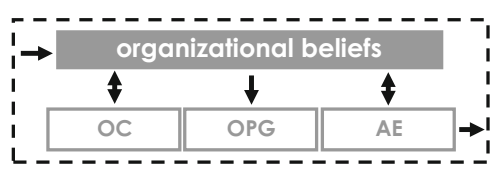

Fig. 4. Reasoning component

Further, in specializing some of the basic concepts (see above) from an agenttechnology perspective (consistent with AORTA), we consider the following:

- Autonomy: agents operate without the direct intervention of humans or others, and have some kind of control of their actions and internal state;

- Social "ability": agents interact with other agents (and possible humans) via some kind of agent communication language;

- Reactivity: agents perceive their environment (which may be the physical world, a user via a graphical user interface, a collection of other agents, the Internet, or perhaps all of those combined), and respond in a timely fashion to changes that occur in it;

- Pro-activeness: agents do not simply act in response to their environment, they are also capable of exhibiting goal-driven behavior, by taking the initiative.

Hence, we define an agent as an entity that is capable of operating autonomously, interacting with other agents, perceiving and reacting to changes in the environment, and taking actions toward solving an objective.

Next to that, it is considered common to have agent systems where an agent represents a larger group of agents; such an agent is often tasked with coordinating the other agents in the group (such tasks can be captured with the notions of role and objective, with agents enacting roles). Thus, by applying the agent concept, we could conveniently capture the notions of autonomy, social ability, reactivity and pro-activeness that we find useful with regard to the challenge of modeling complex (context-aware) systems where it is not straightforward understanding how every system part works in detail.

Finally, we present below the enrichment (from an agent-technology perspective) with regard to the basic concepts that were presented above: 
SYSTEM: A system is represented through the notion of an agent that enacts a designated system role. The role specifies the objectives (obj) of the system, reflecting its purpose. These objectives can be decomposed into sub-objectives by obj-statements. We decompose the objective of the system into sub-objectives, matching the objectives of the sub-systems. We show the dependencies (dep) between the system and its sub-systems by dep-statements. SUB-SYSTEM: We represent individual sub-systems by agents enacting roles, specifying the sub-objectives of the system that they address. We also make explicit the dependency between the system and its sub-systems by dep-statements.

ENVIRONMENT: We represent the parts of the environment that we assume interact with the system by agents. An environmental agent may enact a role, meaning that the system can expect it to carry out a certain objective, but this is not required.

ENTITIES: Inspired by the agent notion as considered in [Wolldr95], we represent entities in general by agents and roles

- human vs artificial entities - no distinction; an agent can represent a human and implement a human behavior model but from a model perspective, human and artificial agents are indistinguishable;

- passive vs autonomous entities; agents that implement proactive behavior models are inherently capable of autonomous behavior, whereas agents that implement reactive behavior models are suited for representing passive entities;

- sensing vs actuator entities; we have roles dedicated to sensing and actuator such that we represent these entities by agents enacting those roles.

ROLE and ACTOR-ROLE: We represent roles and their designated objectives by rolestatements, and the entity-role combination by rea-statements, stating that an agent enacts a role.

ACTOR: We represent an actor by its designated agent.

USER: The user is a specific agent that plays the role of user. For context-aware systems, the system agent depends on the user to deliver its service and for gaining feedback. The user role may have certain objectives but this is not required.

ACTION and INTERACTION: An agent is capable of performing actions, and in the OPGphase, it generates options based on its beliefs. It then deliberates the action to take in the AE-phase. We represent the concept interaction by dep-statements, meaning that multiple agents are collaborating in solving an objective.

OBJECTIVE: We represent objectives primarily by role- and $o b j$ - statements.

REGULATIONS: We represent regulations by condition statements, stating situations where obligations to carry out objectives get activated. In the oc-phase, the agent updates itself for current active obligations and violated obligations with obl- and viol-statements.

VALUES: Values are reflected in the regulations expressed by cond-predicates.

\subsection{Proposed Meta-Model}

After having introduced our concepts (in synch with SDBC), we have derived a meta-model accordingly, considered a key contribution of the current paper. The meta-model is presented in Fig. 5, using the notations of UML - Class Diagram [25].

As it is seen in the figure, we consider a system and its environment. Both are composed of numerous entities which in turn can be components (non proactive) or agents (pro-active and intelligent). One entity (an agent, for example) can enact many different roles (and in this research, we limit ourselves to four role categories, namely: user, sensor, actuator, and processor) that are restricted 
by corresponding rules and are subject of regulations. A regulation in turn is composed of many rules and is affecting not only the roles but the system as a whole.

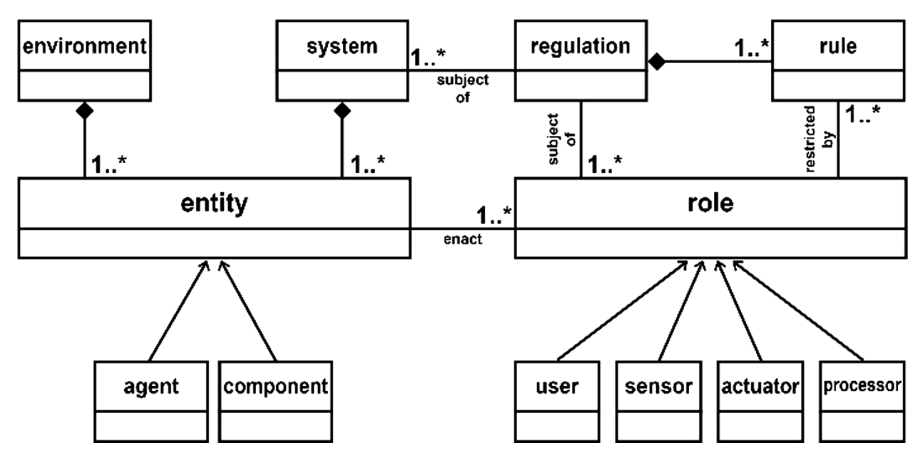

Fig. 5. Proposed meta-model

Since we are taking particularly an agent perspective, we consider it important matching roles to their corresponding executing agents because it is not for sure that anybody would have the right capabilities of fulfilling a role.

With regard to what an agent is capable of doing, AORTA takes a discrete approach to capabilities: the capabilities of an agent are defined as the set of states that an agent can achieve. More concretely, an agent is defined as the tuple ( $\boldsymbol{\alpha}, \mathbf{M S}, \mathbf{A R}, \mathbf{F}, \mathbf{C}, \boldsymbol{\mu})$ where $\boldsymbol{\alpha}$ is the name of the agent, $\mathbf{M S}$ is its "mental" state, $\mathbf{A R}$ are its rules of reasoning, $\mathbf{F}$ is a set of transitioning functions (Action $\times \mathrm{MS} \rightarrow \mathrm{MS}$ ), $\mathbf{C}$ are its capabilities and $\boldsymbol{\mu}$ is a "mailbox" for incoming messages from and outgoing messages to other agents.

When considering a role enactment, an agent checks whether its capabilities overlap the objectives of that role (if not, an enactment would be impossible). One can split a role into two roles where one defines what objectives are expected of an agent enacting the "partial" role and what is expected of an agent enacting the "full" role.

As mentioned in the Introduction, we propose at the end of this section refined guidelines (inspired by SDBC and the meta-model).

\subsection{Refined Design Guidelines}

In following SDBC (see Fig. 3) and implementing the meta-model (see Fig. 5), we propose refined design guidelines, and we limit ourselves only to considering the strengths of agent technology with regard to the three categories of context-aware systems addressed in the current paper.

The three system behavior perspectives (SMCAS, UDCAS, and VSCAS - see Sect. 2) considered in combination, referring to the meta-model, inspire a proposed design vision; according to it, a system uses an AORTA Engine considered for doing all three things simultaneously, as it is illustrated in Fig. 6. 
As the figure suggests, the user is situated in the environment and the system uses sensors to receive info from the environment, including the user, and actuators to manage sub-systems, realizing internal optimizations. The AORTA Engine allows the system to conform to rules and regulations (regulations are encoded, reflecting the values the system should consider). The AORTA Engine checks the input from the Analysis Engine to identify obligated, forbidden or allowed actions, and what organizational influence those actions have (enacting a role involves notifying other entities). The output is provided as input to the Planner that in turn decides the action to be taken.

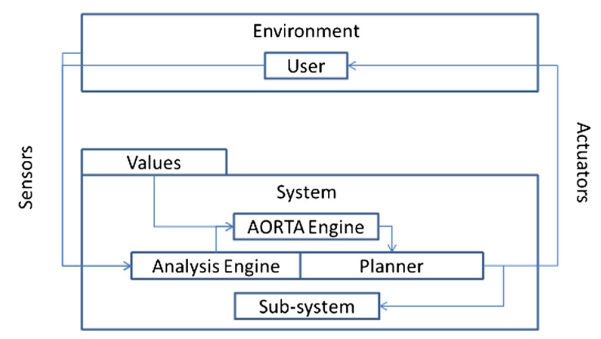

Fig. 6. A system combining features of SMCAS, UDCAS, and VSCAS

\section{The Border Security Case}

Unmanned aircraft (for example, drones) are a way to facilitate the surveillance along land borders, as according to a case example considered in [21].

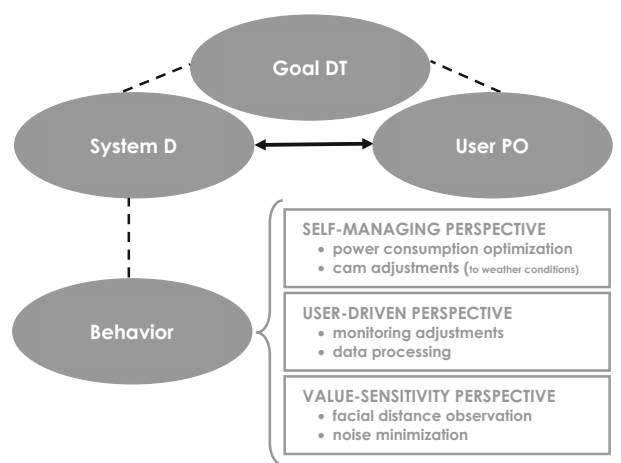

Fig. 7. Exemplifying the SMCAS, UDCAS, and VSCAS perspectives

Drones are capable of carrying and running infrared (and regular) surveillance cameras and based on their input, scarce capacity can be allocated. The goal is to find illegal activities. Despite drones' advantages, there can be some unintended effects: Drones can make noise and scare ordinary people who are passing the borders in a 
legitimate way, often at check-points; custom officials and/or police officers might be scared and/or disgraced by drones. Next to that, (video) recording activities might be violating the privacy of people. Hence, it should be avoided that: (i) drones come too close to legitimate activities; (ii) data that is not needed is recorded and shared. This asks for balancing between the internal objectives of finding illegal activities and the societal demands that are two-fold: it is needed to avoid drones getting too close to people and it is also needed to ensure their privacy.

We hence make the following explicit: (A) We do address SYSTEM D - a drone system, featuring a drone flying over a border and supporting border police officers; (B) System $D$ is delivering services to USER PO - the border police officers who are patrolling the border; (C) The goal in context is: GOAL DT, featuring the detection of illegal activities in general (trespassers, in particular). This is reflected in Fig. 7.

As also seen from the figure, we have identified six case-specific behavior lines, two per each of the system behavior perspectives:

[POWER CONSUMPTION OPTIMIZATION] [SMCAS PERSPECTIVE]: When a drone is flying, it is exchanging information with the ground station - the drone is sending sensor data to the station that is in turn generating instructions. Hence, it is at the ground station where the flight is controlled such that it is guaranteed that the drone mission is completed. This requires that the power consumption is optimized, such that the drone has enough power to complete the mission and get back.

[CAM ADJUSTMENTS] [SMCAS PERSPECTIVE]: When a drone is flying, it counts on its cameras, such that it is capable of adjusting its flight accordingly. Hence, those cameras need to be adjusted when weather conditions change.

[MONITORING ADJUSTMENTS] [UDCAS PERSPECTIVE]: When a drone is flying, it is realizing its mission to support border police officers (the "user") and for this, it should adjust its monitoring to mainly cover those areas that are not close to where police officers are.

[DATA PROCESSING] [USCAS PERSPECTIVE]: When a drone is flying, raw sensor data is processed by the drone itself and/or by the ground station, such that higher-level context information is derived and delivered to border police officers.

[FACIAL DISTANCE OBSERVATION] [VSCAS PERSPECTIVE]: When a drone is flying, it is to act in a privacy-sensitive way, which means that without explicit instructions, the drone should not (video) capture people's facial information or if this happens, the photo/video material should be blurred accordingly.

[NOISE MINIMIZATION] [VSCAS PERSPECTIVE]: When a drone is flying, it should avoid noise-polluting residential environments, which means that if this wouldn't be mission-critical, the drone should avoid approaching residential areas.

Acting adequately with regard to all those perspectives is considered important because it wouldn't be acceptable neither "sacrificing" the drone, nor compromising the mission, nor disregarding values. Nevertheless, it is sometimes impossible to satisfy all this. For example: If from a SMCAS perspective, the drone should immediately turn back (with indications of insufficient power to go on) but from a UDCAS perspective, the drone should go on to approach an area of interest, then what is the solution? Resolving such TENSIONS is considered non-trivial because we argue that "universal rules" cannot work in cases like this. Thus, it is a matter of sophisticated prioritization to come to the "right" solution. If, for example, a border control mission is on and a noise pollution is observed, then if the mission is just a routine surveillance, probably 
the noise-pollution should be avoided but if the mission is targeting trespassers, then the neighborhood silence may be sacrificed and for sure the residents would understand and appreciate such actions. For this reason, the AORTA drone mission modeling is to be complemented by a "prioritization scheme" which is not shown in the current section, for the sake of brevity.

What we are demonstrating is the OVERALL model (simplified to what Fig. 7 suggests) where all three perspectives are "super-imposed" (such that tensions could be straightforwardly identified) - this is presented in Table 1.

Table 1. Exemplifying the overall behavior, by applying AORTA.

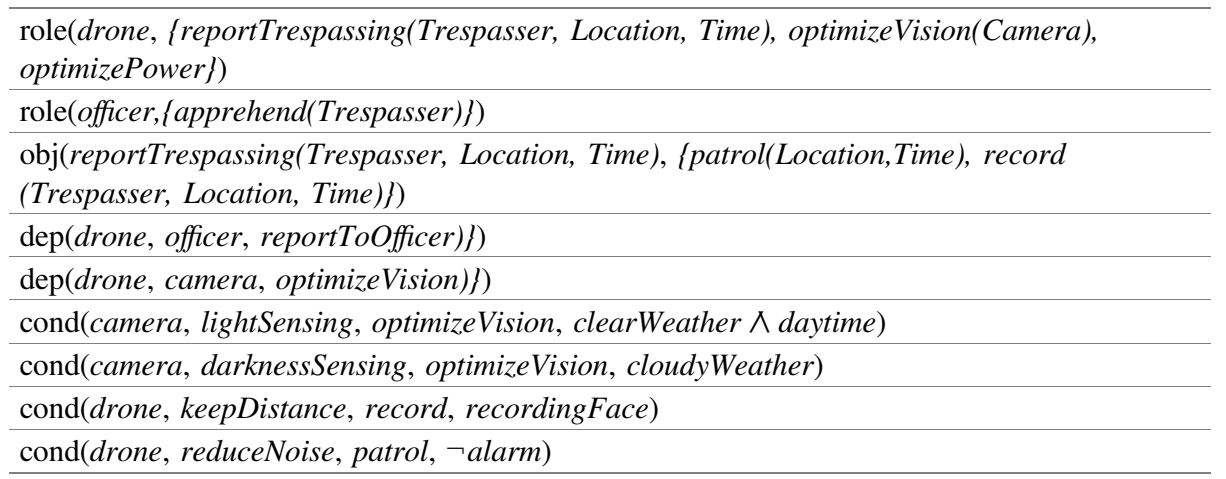

\section{Related Work}

AORTA is based upon the OperA meta-model for agent organizations. MOISE+ [26] is another meta-model for agent organizations that is well known in AI (Artificial Intelligence)-communities, and which is implemented in the agent-programming platform JaCaMo [2]. With regard to agent-based AI systems: "Pepper" is a robot working together with humans in a socially acceptable way. Pepper perceives its environment through visual sensors and can also receive input through a touch screen. Pepper is able to make simple gestures, speak, and move around on wheels. Pepper's behavior is programmed using the agent-based language GOAL [9] in which an agent is specified in terms of a mental state with personal info. In contrast, we propose using AORTA in which an agent maintains personal information and organizational information separately. This relates to user-driven context-aware systems where for example Body-Area Networks are implemented to capture vital signs from the body of a patient from distance, such that this information is processed and communicated in an intelligent way [1] while self-managing systems were widely used in home appliances that adjust their operation with regard to some goal that concerns a unit, such as neighborhood [24]. This all concerns values that are abstract and non-functional and that is how they are considered to date [7], not aligning them adequately with the processes that concern the design of software. We believe that the current work is a step forward in approaching methodologically context-aware systems, in general and particularly - the three categories of context-aware systems addressed in this paper. 


\section{Conclusions}

Furthering previous research that touches upon the enterprise-modeling-driven software specification, we have particularly addressed context-aware (software) systems in the current paper. We have identified and studied three categories of context-aware systems, featuring context-based system behavior adaptations that concern the optimization of system-internal processes, the maximization of the user-perceived effectiveness, and the consideration of relevant public values. Super-imposing those three system behavior perspectives and in synch with previous work (the SDBC approach), we have identified concepts and we have enriched them from an agent-technology perspective (in line with the AORTA framework), reflecting this in a meta-model that is considered a major contribution of the paper. Inspired by the meta-model, we have provided a partial refinement of the SDBC design guidelines, taking an agent technology perspective and focusing on the three above-mentioned system behavior perspectives, and we have partially illustrated this by means of a case example featuring land border security. As future work we plan to CONSOLIDATE our SDBC-AORTA-driven proposal into one dedicated design approach that is specific to context-aware systems.

Acknowledgements. This work is supported by: (i) the TU Delft - Delft Pilot project; (ii) Technical University of Denmark and the PDC A/S project. We would like to thank Jeroen van den Hoven for his support and guidance.

\section{References}

1. AWARENESS: Freeband AWARENESS Project (2008). http://www.freeband.nl

2. Boissier, O., Bordini, R.H., Hübner, J.F., Ricci, A., Santi, A.: Multi-agent oriented programming with JaCaMo. Sci. Comput. Program. 78, 6 (2013)

3. Brun, Y., Di Marzo Serugendo, G., Gacek, C., Giese, H., Kienle, H., Litoiu, M., Müller, H., Pezzè, M., Shaw, M.: Engineering self-adaptive systems through feedback loops. In: Cheng, Betty H.C., de Lemos, R., Giese, H., Inverardi, P., Magee, J. (eds.) Software Engineering for Self-Adaptive Systems. LNCS, vol. 5525, pp. 48-70. Springer, Heidelberg (2009). https:// doi.org/10.1007/978-3-642-02161-9_3

4. Cockburn, A.: Writing Effective Use Cases. Addison-Wesley, Boston (2000)

5. Dey, A.K.: Understanding and using context. Pers. Ubiquit. Comput. 5(1), 4-7 (2001)

6. Dignum, V.: A model for organizational interaction: based on agents, founded in logic. $\mathrm{Ph}$. D. thesis, Utrecht University (2004)

7. Friedman, B., Hendry, D.G., Borning, A.: A survey of value sensitive design methods. In: A Survey of Value Sensitive Design Methods, Foundations and Trends, vol. 1, p. 76 (2017)

8. Google Dictionary: The website of Google Dictionary (2018). http://www.google.com

9. Hindriks, K.V.: Programming rational agents in GOAL. In: El Fallah Seghrouchni, A., Dix, J., Dastani, M., Bordini, R.H. (eds.) Multi-Agent Programming: Languages, pp. 119-157. Springer, Tools and Applications (2009). https://doi.org/10.1007/978-0-387-89299-3_4

10. Huebscher, M.C., McCann, J.A.: A survey of autonomic computing - degrees, models, and applications. ACM Comput. Surv. 40(3) (2008). Article no. 7 
11. Jensen, A.S., Dignum, V., Villadsen, J.: A framework for organization-aware agents. Auton. Agent. Multi-Agent Syst. 31(3), 387-422 (2017)

12. Kephart, J.O., Chess, D.M.: The vision of autonomic computing. Computer 36(1), 41-50 (2003)

13. Kruchten, P.: The Rational Unified Process: An Introduction. Addison-Wesley, Boston (2003)

14. Mahdavi-Hezavehi, S., Avgeriou, P., Weyns, D.: A classification framework of uncertainty in architecture-based self-adaptive systems with multiple quality requirements. In: Mistrik, I., Ali, N., Kazman, R., Grundy, J., Schmerl, B. (eds.) Managing Trade-offs in Adaptable Software Architectures, 1st edn. Elseiver Inc. (2016)

15. MDA: The OMG Model Driven Architecture (2018). http://www.omg.org/mda

16. Muehl, G., Werner, M., Jaeger, M.A., Herrmann, K., Parzyjegla, H.: On the definitions of self-managing and self-organizing systems. In: ITG/GI Symposium on Communication in Distributed Systems - 15, Bern, Switzerland, pp. 1-11 (2007)

17. Shishkov, B.: Enterprise Information Systems, A Modeling Approach. IICREST, Sofia (2017)

18. Shishkov, B., Janssen, M.: Enforcing context-awareness and privacy-by-design in the specification of information systems. In: Shishkov, B. (ed.) BMSD 2017. LNBIP, vol. 309, pp. 87-111. Springer, Cham (2018). https://doi.org/10.1007/978-3-319-78428-1_5

19. Shishkov, B., Mendling, J.: Business process variability and public values. In: Shishkov, B. (ed.) BMSD 2018. LNBIP, vol. 319, pp. 401-411. Springer, Cham (2018)

20. Shishkov, B., Janssen, M., Yin, Y.: Towards context-aware and privacy-sensitive systems. In: BMSD 2017, 7th International Symposium on Business Modeling and Software Design. SCITEPRESS (2017)

21. Shishkov, B., Mitrakos, D.: Towards context-aware border security control. In: 6th International Symposium on Business Modeling and Software Design, BMSD 2016. SCITEPRESS (2016)

22. Shishkov, B., van Sinderen, M.: From user context states to context-aware applications. In: Filipe, J., Cordeiro, J., Cardoso, J. (eds.) ICEIS 2007. LNBIP, vol. 12, pp. 225-239. Springer, Heidelberg (2008). https://doi.org/10.1007/978-3-540-88710-2_18

23. Shishkov, B., Van Sinderen, M.J., Quartel, D.: SOA-driven business-software alignment. In: IEEE International Conference on e-Business Engineering, ICEBE 2006. IEEE (2006)

24. Shishkov, B., Warnier, M., Van Sinderen, M.: On the application of autonomic and context-aware computing to support home energy management. In: Proceedings of ICEIS 2010 - the 12th International Conference on Enterprise Information Systems, Funchal, PT, 8-12 June 2010. SCITEPRESS, Setúbal (2010)

25. UML: The Unified Modeling Language (2018). http://www.uml.org

26. van Riemsdijk, M.B., Hindriks, K., Jonker, C.: Programming organization-aware agents. In: Aldewereld, H., Dignum, V., Picard, G. (eds.) ESAW 2009. LNCS (LNAI), vol. 5881, pp. 98-112. Springer, Heidelberg (2009). https://doi.org/10.1007/978-3-642-10203-5_9

27. Wooldridge, M., Jennings, N.R.: Intelligent agents: theory and practice. Knowl. Eng. Rev. 10(2), 115-152 (1995) 